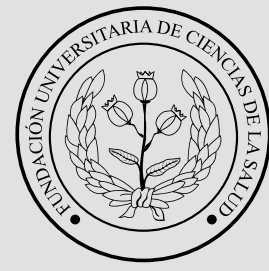

FUCS
Rere 10

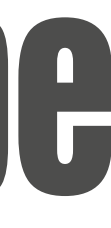

\title{
Valoración de la incidencia del COVID-19 en Colombia para el año 2020
}

\section{Evaluation of the incidence of COVID-19 in Colombia in year 2020}

\footnotetext{
${ }^{a}$ Ingeniero. Magister en Gestión de la Tecnología Educativa, Especialista en Administración de la Informática Educativa. Docente de matemáticas e Investigador, Secretaría de Educación de Soacha, Cundinamarca.
}

\section{R ES U M EN}

Introducción: la enfermedad por coronavirus humano 2019 (COVID-19) apareció a fines de diciembre de 2019 y un nuevo betacoronavirus después denominado síndrome respiratorio agudo severo coronavirus-2 (SARS-CoV-2), indicó ser la causa. Este virus podría transmitirse cómodamente de persona a persona y propagarse rápido por todo el mundo. Objetivo: estimar la frecuencia de incidencia, de marzo hasta diciembre de 2020 en los departamentos de Colombia para COVID-19. Metodología: para desarrollar la investigación se utilizó la base de datos de las personas contagiadas por COVID-19, la información de los datos corresponde al período acumulado entre 6 de marzo de 2020 a 31 de diciembre de 2020 para los departamentos de Colombia. Resultados: se puntualizó que aquellos con mayor incidencia acumulada (IA) de casos positivos por COVID-19 están en Bogotá 6,084\%, Quindío 4,257\%, Antioquía 3,917\%, San Andrés 3,487\%, Caquetá 3,654\% y Atlántico 3,452\%. Los departamentos con menor IA son: Arauca 1,555\%, Magdalena 1,493\%, Guajira 1,466\%, Cauca 1,254\% y Vichada 1,018\%. Conclusiones: en los 33 departamentos de Colombia la frecuencia de casos, incidencia acumulada, fue heterogénea. Estos resultados contribuyen a comprender el comportamiento epidemiológico de la enfermedad en todos los grupos etarios de la población en los departamentos incluidos en este estudio.

Palabras clave: SARS-CoV-2, COVID-19, pandemia, incidencia.

(C) 2021 Fundación Universitaria de Ciencias de la Salud - FUCS Este es un artículo Open Access bajo la licencia CC BY-NC-ND (http://creativecommons.org/licenses/by-nc-nd/4.0/).

INFORMACIÓN DEL ARTÍCULO

Historia del artículo:

Fecha recibido: enero 26 de 2021 Fecha aceptado: febrero 2 de 2021
Autor para correspondencia.

Ing. Jorge Enrique Díaz Pinzón jediazp@unal.edu.co
DOI

10.31260/RepertMedCir.01217372.1174 
Introduction: human coronavirus disease 2019 (COVID-19) emerged in late December 2019. A novel beta coronavirus, later named severe acute respiratory syndrome coronavirus-2 (SARS-CoV-2), was identified to be the cause. This virus could easily be transmitted from person to person and spread rapidly around the world. Objective: to estimate the incidence rate for COVID-19, from March to December 2020 in each department in Colombia. Methodology: this research was developed using the database of people infected by COVID-19 with the information collected over the period between March 62020 and December 312020 in each department in Colombia. Results: the highest cumulative incidence (CI) of confirmed COVID-19 cases was reported in Bogota $6.084 \%$, Quindío 4.257\%, Antioquia 3.917\%, San Andres 3.487\%, Caqueta 3.654\% and Atlantico $3.452 \%$. The departments with the lowest CI are: Arauca 1.555\%, Magdalena $1.493 \%$, Guajira 1.466\%, Cauca $1.254 \%$ and Vichada $1.018 \%$. Conclusions: the frequency of cases, cumulative incidence, across the 33 departments of Colombia, was heterogeneous. These results contribute to understand the epidemiological behavior of the disease in all age groups of the population of the departments included in this study.

Key words: SARS-CoV-2, COVID-19, pandemic, incidence.

(C) 2021 Fundación Universitaria de Ciencias de la Salud - FUCS. This is an open access article under the CC BY-NC-ND license (http://creativecommons.org/licenses/by-nc-nd/4.0/).

INTRODUCCIÓN

La enfermedad por coronavirus humano 2019 (COVID-19) apareció a finales de diciembre de $2019^{1,2}$ y un nuevo betacoronavirus, luego denominado síndrome respiratorio agudo severo coronavirus-2 (SARS-CoV-2), fue la causa. Este virus podría transmitirse de persona a persona y propagarse con rapidez por todo el mundo. ${ }^{3,4}$ Además, se está diseminando en más de 210 países y territorios a nivel mundial. ${ }^{5-7}$ La alta tasa de infección del SARS-CoV-2 conduce a su rápida propagación. ${ }^{8}$ Estudios recientes han informado un progreso significativo en el desarrollo de la terapia COVID-19 y vacunas basadas en la proteína $\mathrm{S}$ o RBD. ${ }^{9-11}$

Se han reconocido muchos casos en todos los continentes y el 6 de marzo 2020 se confirmó el primero en Colombia. La infección se evidencia cuando una persona enferma tose o estornuda y expulsa partículas del virus que entran en contacto con otras personas. ${ }^{12}$

En epidemiología, el desarrollo de la investigación es análogo al utilizado en el resto de las ciencias. Cuando se investiga la salud de la población asimismo se proponen una o varias explicaciones hipotéticas que después son reducidas a contrastación empírica. En este proceso los conceptos de medición y de variable trascienden primordiales. ${ }^{13}$

En epidemiología, una de las medidas de frecuencia de enfermedad más utilizadas es la incidencia ${ }^{14}$ que se define como el número de casos nuevos de una enfermedad que se desarrollan en una población durante un período de tiempo determinado. ${ }^{15-17} \mathrm{Su}$ cálculo se estima mediante:

Número de casos nuevos de una enfermedad

Incidencia $=$ x 100
"Como todas las proporciones, la incidencia no tiene dimensión y nunca toma valores menores de 0 ó mayores de 1, siendo frecuente expresarla en términos de porcentaje, en tanto por ciento tanto por mil, en función de la rareza de la enfermedad estudiada". ${ }^{14}$

La incidencia acumulada (IA) es la proporción de individuos sanos que contraen la enfermedad a lo largo de un período de tiempo determinado. ${ }^{14}$ Se calcula según:

Número de casos nuevos de una enfermedad durante el seguimiento

Incidencia $=$ Total de población en riesgo al inicio x 100 del seguimiento

La incidencia acumulada suministra una estimación de la probabilidad o el riesgo de que un individuo libre de una explícita enfermedad la desarrolle durante un período detallado de tiempo. Como cualquier proporción, suele venir dada en términos de porcentaje. Además, al no ser una tasa, es indispensable que se acompañe del periodo de observación para poder ser dilucidada. ${ }^{15,16}$

\section{METODOLOGÍA}

La investigación se efectuó mediante un enfoque cuantitativo que es aquella en la que se acumulan y examinan datos cuantitativos sobre variables y estudia las propiedades y fenómenos cuantitativos, según Hurtado y Toro (1998) citado por Díaz. ${ }^{17}$ 


\section{Población}

Esta investigación se centró en la población de contagiados por COVID-19 en Colombia, con la información proveniente del Instituto Nacional de Salud ${ }^{18}$, en el periodo comprendido entre 6 de marzo de 2020 a 31 de diciembre 2020 y la población proyectada para 2020 por parte del DANE $^{19}$ (tabla 1).

Tabla 1. Población y casos nuevos por COVID-19 en Colombia a 31 de diciembre 2020

\begin{tabular}{|c|c|c|}
\hline Mes & Población & Casos nuevos \\
\hline Marzo & 50372424 & 906 \\
\hline Abril & 50371518 & 5601 \\
Mayo & 50365917 & 22876 \\
Junio & 50343041 & 68463 \\
Julio & 50274578 & 197662 \\
Agosto & 50076916 & 319660 \\
Septiembre & 49757256 & 214511 \\
Octubre & 49542745 & 244505 \\
Noviembre & 49298240 & 242622 \\
Diciembre & 49055618 & 325969 \\
\hline
\end{tabular}

\section{RESULTADOS}

\section{Cálculo de la incidencia por contagio por COVID-19 para 2020}

Para determinar la incidencia por contagio por COVID-19 para 2020 se procedió de la siguiente manera. Con los datos de la tabla 1, por ejemplo, del mes de marzo se tomaron los casos nuevos por COVID-19, en este caso 906, y los dividimos entre la población en riesgo para ese mes (50'372.424), y este resultado lo multiplicamos por 100, por tanto, la incidencia para el mes de marzo fue de $0,001 \%$. Para el mes de abril se restó la población del mes de marzo con los casos nuevos el mismo mes, luego se dividieron los casos nuevos de abril y los dividimos con la nueva población para abril y el resultado lo multiplicamos por 100, así sucesivamente hasta llegar al mes de diciembre 2020. En la figura 1 se aprecian los datos de la incidencia en porcentaje $(\%)$, para cada uno de los meses de marzo a diciembre 2020 en Colombia, se detalla que los meses con mayor incidencia de casos positivos fueron diciembre $0,664 \%$, agosto $0,638 \%$, octubre $0,492 \%$ y septiembre $0,431 \%$; y los meses con menor incidencia fueron: julio 0,393\%, junio 0,135\%, mayo 0,04\%, abril 0,011\% y marzo $0,001 \%$.

Fuente. El autor.

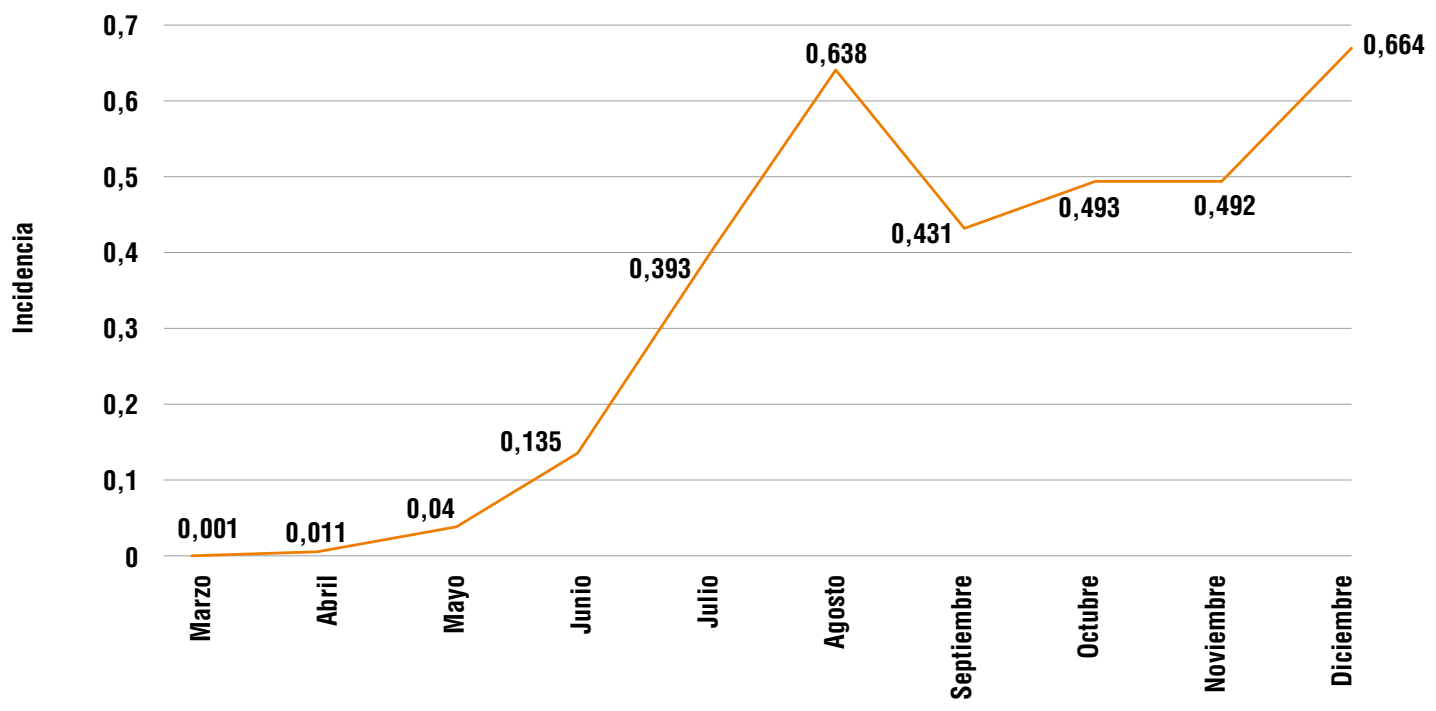

Año 2020

Figura 1. Incidencia en porcentaje de contagio por COVID-19 para 2020. Fuente: El autor. 
Tabla 2. Casos nuevos por departamentos y meses para COVID-19 en Colombia

\begin{tabular}{|c|c|c|c|c|c|c|c|c|c|c|}
\hline Departamentos & Mar. & Abr. & May. & Jun. & Jul. & Ago. & Sep. & Oct. & Nov. & Dic. \\
\hline Amazonas & 0 & 104 & 101 & 450 & 209 & 191 & 40 & 165 & 241 & 96 \\
\hline Antioquia & 101 & 331 & 672 & 3329 & 28334 & 48220 & 34189 & 54240 & 44512 & 47664 \\
\hline Arauca & 0 & 0 & 1 & 75 & 138 & 711 & 800 & 1621 & 730 & 500 \\
\hline Atlántico & 33 & 280 & 3621 & 19435 & 28945 & 11778 & 3435 & 4326 & 6266 & 15854 \\
\hline Bogotá & 383 & 2229 & 7394 & 20027 & 71960 & 109366 & 57828 & 52469 & 53653 & 95815 \\
\hline Bolívar & 42 & 252 & 2897 & 5943 & 9107 & 7033 & 3588 & 4138 & 5455 & 13343 \\
\hline Boyacá & 6 & 33 & 172 & 157 & 650 & 2791 & 3532 & 7078 & 7276 & 6888 \\
\hline Caldas & 15 & 54 & 65 & 125 & 696 & 1989 & 3007 & 7873 & 11344 & 7361 \\
\hline Caquetá & 0 & 8 & 16 & 11 & 902 & 5136 & 2413 & 2845 & 2057 & 1612 \\
\hline Casanare & 1 & 17 & 17 & 31 & 241 & 752 & 1380 & 2630 & 1893 & 1816 \\
\hline Cauca & 9 & 29 & 66 & 271 & 1549 & 3363 & 4208 & 3772 & 2529 & 2910 \\
\hline Cesar & 11 & 44 & 198 & 772 & 2075 & 7710 & 9208 & 5951 & 3812 & 3776 \\
\hline Chocó & 0 & 13 & 213 & 1317 & 1451 & 727 & 274 & 198 & 331 & 354 \\
\hline Córdoba & 2 & 24 & 98 & 669 & 4870 & 14275 & 4162 & 2223 & 1904 & 1349 \\
\hline Cundinamarca & 44 & 199 & 588 & 1859 & 5795 & 14597 & 10416 & 8930 & 10078 & 14683 \\
\hline Guainía & 0 & 0 & 6 & 8 & 0 & 125 & 733 & 260 & 72 & 30 \\
\hline Guajira & 1 & 8 & 53 & 392 & 1657 & 3033 & 2995 & 1925 & 1858 & 2231 \\
\hline Guaviare & 0 & 0 & 0 & 37 & 79 & 157 & 531 & 663 & 368 & 128 \\
\hline Huila & 24 & 96 & 129 & 91 & 551 & 4275 & 6579 & 10898 & 7451 & 4786 \\
\hline Magdalena & 10 & 192 & 411 & 1239 & 4303 & 6310 & 2891 & 1958 & 2926 & 1061 \\
\hline Meta & 11 & 360 & 604 & 276 & 1505 & 6209 & 7274 & 6654 & 5488 & 5572 \\
\hline Nariño & 4 & 114 & 984 & 2341 & 4523 & 6107 & 4241 & 3566 & 3654 & 5774 \\
\hline Norte de Santander & 19 & 50 & 52 & 207 & 2007 & 9509 & 3952 & 5542 & 7627 & 7805 \\
\hline Putumayo & 0 & 0 & 9 & 19 & 895 & 1977 & 894 & 601 & 591 & 894 \\
\hline Quindío & 16 & 43 & 52 & 56 & 180 & 992 & 2432 & 6255 & 7152 & 6466 \\
\hline Risaralda & 34 & 165 & 56 & 256 & 1123 & 4568 & 5035 & 6135 & 6119 & 10722 \\
\hline San Andrés & 1 & 5 & 11 & 6 & 14 & 181 & 1256 & 409 & 259 & 323 \\
\hline Santander & 11 & 29 & 36 & 643 & 3039 & 14464 & 13062 & 11051 & 10998 & 17360 \\
\hline Sucre & 1 & 0 & 20 & 1226 & 4532 & 5780 & 2369 & 1506 & 1077 & 949 \\
\hline Tolima & 9 & 59 & 195 & 673 & 1630 & 4656 & 5168 & 7410 & 10022 & 14318 \\
\hline Valle & 117 & 824 & 2525 & 6505 & 14669 & 22306 & 15756 & 20649 & 24715 & 29803 \\
\hline Vaupés & 0 & 0 & 11 & 17 & 33 & 279 & 429 & 295 & 55 & 22 \\
\hline Vichada & 0 & 0 & 1 & 0 & 1 & 92 & 433 & 267 & 125 & 231 \\
\hline
\end{tabular}

Fuente. El autor.

En la tabla 2 se aprecian los casos nuevos por COVID-19 por departamentos en Colombia para los meses de marzo a diciembre 2020.

En la tabla 3 se observa la población por departamentos en Colombia y en los meses de marzo a diciembre 2020.
A partir de abril, se restan los casos nuevos del mes de marzo (tabla 2), por eso disminuye la población mes a mes, se sigue el mismo procedimiento hasta llegar al mes de diciembre. 
Tabla 3. Población por departamentos en Colombia a 31 de diciembre 2020

\begin{tabular}{|c|c|c|c|c|c|c|c|c|c|c|}
\hline Departamentos & Mar. & Abr. & May. & Jun. & Jul. & Ago. & Sep. & Oct. & Nov. & Dic. \\
\hline Amazonas & 79020 & 78916 & 78815 & 78365 & 78156 & 77965 & 77925 & 77760 & 77519 & 77423 \\
\hline Antioquia & 6677930 & 6677599 & 6676927 & 6673598 & 6645264 & 6597044 & 6562855 & 6508615 & 6464103 & 6416439 \\
\hline Arauca & 294206 & 294206 & 294205 & 294130 & 293992 & 293281 & 292481 & 290860 & 290130 & 289630 \\
\hline Atlántico & 2722128 & 2721848 & 2718227 & 2698792 & 2669847 & 2658069 & 2654634 & 2650308 & 2644042 & 2628188 \\
\hline Bogotá & 7743955 & 7741726 & 7734332 & 7714305 & 7642345 & 7532979 & 7475151 & 7422682 & 7369029 & 7273214 \\
\hline Bolívar & 2180976 & 2180724 & 2177827 & 2171884 & 2162777 & 2155744 & 2152156 & 2148018 & 2142563 & 2129220 \\
\hline Boyacá & 1242731 & 1242698 & 1242526 & 1242369 & 1241719 & 1238928 & 1235396 & 1228318 & 1221042 & 1214154 \\
\hline Caldas & 1018453 & 1018399 & 1018334 & 1018209 & 1017513 & 1015524 & 1012517 & 1004644 & 993300 & 985939 \\
\hline Caquetá & 410521 & 410513 & 410497 & 410486 & 409584 & 404448 & 402035 & 399190 & 397133 & 395521 \\
\hline Casanare & 435195 & 435178 & 435161 & 435130 & 434889 & 434137 & 432757 & 430127 & 428234 & 426418 \\
\hline Cauca & 1491937 & 1491908 & 1491842 & 1491571 & 1490022 & 1486659 & 1482451 & 1478679 & 1476150 & 1473240 \\
\hline Cesar & 1295387 & 1295343 & 1295145 & 1294373 & 1292298 & 1284588 & 1275380 & 1269429 & 1265617 & 1261841 \\
\hline Choco & 544764 & 544751 & 544538 & 543221 & 541770 & 541043 & 540769 & 540571 & 540240 & 539886 \\
\hline Córdoba & 6677930 & 6677906 & 6677808 & 6677139 & 6672269 & 6657994 & 6653832 & 6651609 & 6649705 & 6648356 \\
\hline Cundinamarca & 3242999 & 3242800 & 3242212 & 3240353 & 3234558 & 3219961 & 3209545 & 3200615 & 3190537 & 3175854 \\
\hline Guainía & 50636 & 50636 & 50630 & 50622 & 50622 & 50497 & 49764 & 49504 & 49432 & 49402 \\
\hline Guajira & 965718 & 965710 & 965657 & 965265 & 963608 & 960575 & 957580 & 955655 & 953797 & 951566 \\
\hline Guaviare & 86657 & 86657 & 86657 & 86620 & 86541 & 86384 & 85853 & 85190 & 84822 & 84694 \\
\hline Huila & 1122622 & 1122526 & 1122397 & 1122306 & 1121755 & 1117480 & 1110901 & 1100003 & 1092552 & 1087766 \\
\hline Magdalena & 1427026 & 1426834 & 1426423 & 1425184 & 1420881 & 1414571 & 1411680 & 1409722 & 1406796 & 1405735 \\
\hline Meta & 1063454 & 1063094 & 1062490 & 1062214 & 1060709 & 1054500 & 1047226 & 1040572 & 1035084 & 1029512 \\
\hline Nariño & 1627589 & 1627475 & 1626491 & 1624150 & 1619627 & 1613520 & 1609279 & 1605713 & 1602059 & 1596285 \\
\hline Norte de Santander & 1620318 & 1620268 & 1620216 & 1620009 & 1618002 & 1608493 & 1604541 & 1598999 & 1591372 & 1583567 \\
\hline Putumayo & 359127 & 359127 & 359118 & 359099 & 358204 & 356227 & 355333 & 354732 & 354141 & 353247 \\
\hline Quindío & 555401 & 555358 & 555306 & 555250 & 555070 & 554078 & 551646 & 545391 & 538239 & 531773 \\
\hline Risaralda & 961055 & 960890 & 960834 & 960578 & 959455 & 954887 & 949852 & 943717 & 937598 & 926876 \\
\hline San Andrés & 63692 & 63687 & 63676 & 63670 & 63656 & 63475 & 62219 & 61810 & 61551 & 61228 \\
\hline Santander & 2280908 & 2280879 & 2280843 & 2280200 & 2277161 & 2262697 & 2249635 & 2238584 & 2227586 & 2210226 \\
\hline Sucre & 949252 & 949252 & 949232 & 948006 & 943474 & 937694 & 935325 & 933819 & 932742 & 931793 \\
\hline Tolima & 1339998 & 1339939 & 1339744 & 1339071 & 1337441 & 1332785 & 1327617 & 1320207 & 1310185 & 1295867 \\
\hline Valle & 4532152 & 4531328 & 4528803 & 4522298 & 4507629 & 4485323 & 4469567 & 4448918 & 4424203 & 4394400 \\
\hline Vaupés & 44712 & 44712 & 44701 & 44684 & 44651 & 44372 & 43943 & 43648 & 43593 & 43571 \\
\hline Vichada & 112958 & 112958 & 112957 & 112957 & 112956 & 112864 & 112431 & 112164 & 112039 & 111808 \\
\hline
\end{tabular}

Fuente. El autor. 
Tabla 4. Incidencia por departamentos en Colombia para COVID-19

\begin{tabular}{|c|c|c|c|c|c|c|c|c|c|c|}
\hline Departamentos & Mar. & Abr. & May. & Jun. & Jul. & Ago. & Sep. & Oet. & Nov. & Dic. \\
\hline Amazonas & 0 & 0,132 & 0,128 & 0,5742 & 0,267 & 0,245 & 0,051 & 0,212 & 0,311 & 0,124 \\
\hline Antioquia & 0,001 & 0,005 & 0,010 & 0,0499 & 0,426 & 0,731 & 0,521 & 0,833 & 0,689 & 0,743 \\
\hline Arauca & 0 & 0,000 & 0,000 & 0,0255 & 0,047 & 0,242 & 0,274 & 0,557 & 0,252 & 0,173 \\
\hline Atlántico & 0,001 & 0,010 & 0,133 & 0,7201 & 1,084 & 0,443 & 0,129 & 0,163 & 0,237 & 0,603 \\
\hline Bogotá & 0,004 & 0,029 & 0,096 & 0,2596 & 0,942 & 1,452 & 0,774 & 0,707 & 0,728 & 1,317 \\
\hline Bolívar & 0,001 & 0,012 & 0,133 & 0,2736 & 0,421 & 0,326 & 0,167 & 0,193 & 0,255 & 0,627 \\
\hline Boyacá & 0,000 & 0,003 & 0,014 & 0,0126 & 0,052 & 0,225 & 0,286 & 0,576 & 0,596 & 0,567 \\
\hline Caldas & 0,001 & 0,005 & 0,006 & 0,0123 & 0,068 & 0,196 & 0,297 & 0,784 & 1,142 & 0,747 \\
\hline Caquetá & 0 & 0,002 & 0,004 & 0,0027 & 0,220 & 1,270 & 0,600 & 0,713 & 0,518 & 0,408 \\
\hline Casanare & 0,000 & 0,004 & 0,004 & 0,0071 & 0,055 & 0,173 & 0,319 & 0,611 & 0,442 & 0,426 \\
\hline Cauca & 0,000 & 0,002 & 0,004 & 0,0182 & 0,104 & 0,226 & 0,284 & 0,255 & 0,171 & 0,198 \\
\hline Cesar & 0,000 & 0,003 & 0,015 & 0,0596 & 0,161 & 0,600 & 0,722 & 0,469 & 0,301 & 0,299 \\
\hline Chocó & 0 & 0,002 & 0,039 & 0,2424 & 0,268 & 0,134 & 0,051 & 0,037 & 0,061 & 0,066 \\
\hline Córdoba & 0 & 0,000 & 0,001 & 0,0100 & 0,073 & 0,214 & 0,063 & 0,033 & 0,029 & 0,020 \\
\hline Cundinamarca & 0,001 & 0,006 & 0,018 & 0,0574 & 0,179 & 0,453 & 0,325 & 0,279 & 0,316 & 0,462 \\
\hline Guainía & 0 & 0,000 & 0,012 & 0,0158 & 0,000 & 0,248 & 1,473 & 0,525 & 0,146 & 0,061 \\
\hline Guajira & 0,000 & 0,001 & 0,005 & 0,0406 & 0,172 & 0,316 & 0,313 & 0,201 & 0,195 & 0,234 \\
\hline Guaviare & 0 & 0,000 & 0,000 & 0,0427 & 0,091 & 0,182 & 0,618 & 0,778 & 0,434 & 0,151 \\
\hline Huila & 0,002 & 0,009 & 0,011 & 0,0081 & 0,049 & 0,383 & 0,592 & 0,991 & 0,682 & 0,440 \\
\hline Magdalena & 0,000 & 0,013 & 0,029 & 0,0869 & 0,303 & 0,446 & 0,205 & 0,139 & 0,208 & 0,075 \\
\hline Meta & 0,001 & 0,034 & 0,057 & 0,0260 & 0,142 & 0,589 & 0,695 & 0,639 & 0,530 & 0,541 \\
\hline Nariño & 0,000 & 0,007 & 0,060 & 0,1441 & 0,279 & 0,378 & 0,264 & 0,222 & 0,228 & 0,362 \\
\hline Norte de Santander & 0,001 & 0,003 & 0,003 & 0,0128 & 0,124 & 0,591 & 0,246 & 0,347 & 0,479 & 0,493 \\
\hline Putumayo & 0 & 0,000 & 0,003 & 0,0053 & 0,250 & 0,555 & 0,252 & 0,169 & 0,167 & 0,253 \\
\hline Quindío & 0,002 & 0,008 & 0,009 & 0,0101 & 0,032 & 0,179 & 0,441 & 1,147 & 1,329 & 1,216 \\
\hline Risaralda & 0,003 & 0,017 & 0,006 & 0,0267 & 0,117 & 0,478 & 0,530 & 0,650 & 0,653 & 1,157 \\
\hline San Andrés & 0,001 & 0,008 & 0,017 & 0,0094 & 0,022 & 0,285 & 2,019 & 0,662 & 0,421 & 0,528 \\
\hline Santander & 0,000 & 0,001 & 0,002 & 0,0282 & 0,133 & 0,639 & 0,581 & 0,494 & 0,494 & 0,785 \\
\hline Sucre & 0,000 & 0,000 & 0,002 & 0,1293 & 0,480 & 0,616 & 0,253 & 0,161 & 0,115 & 0,102 \\
\hline Tolima & 0,000 & 0,004 & 0,015 & 0,0503 & 0,122 & 0,349 & 0,389 & 0,561 & 0,765 & 1,105 \\
\hline Valle & 0,002 & 0,018 & 0,056 & 0,1438 & 0,325 & 0,497 & 0,353 & 0,464 & 0,559 & 0,678 \\
\hline Vaupés & 0,000 & 0,000 & 0,025 & 0,0380 & 0,074 & 0,629 & 0,976 & 0,676 & 0,126 & 0,050 \\
\hline Vichada & 0,000 & 0,000 & 0,001 & 0,0000 & 0,001 & 0,082 & 0,385 & 0,238 & 0,112 & 0,207 \\
\hline
\end{tabular}

Fuente. El autor. 
Cálculo de la incidencia de contagio por COVID-19 por departamentos en Colombia

Para determinar por meses la incidencia para el contagio por COVID-19 en los departamentos de Colombia para 2020 (tabla 4), se procedió de la siguiente manera. Con los datos de la tabla 2, por ejemplo, en el caso de Bogotá en el mes de marzo (383), se dividieron los casos nuevos por COVID-19 entre la población en riesgo (7'743.955) de la tabla 3 para ese mes, y este resultado lo multiplicamos por 100, obteniendo como resultado una incidencia para el mes de marzo 2020 en Bogotá de 0,004\%. Para el mes de abril, se restó la población con los casos nuevos de marzo, obteniendo una población de 7'741.726, luego se dividieron los casos nuevos de abril (2.229) y lo dividimos por la nueva población en riesgo (7'741.726), (tabla 3) y el resultado lo multiplicamos por 100, obteniendo una incidencia para el mes de abril de 0,029 y así sucesivamente hasta llegar al mes de diciembre 2020. En la tabla 4 se estiman los datos de la incidencia en porcentaje (\%), para cada uno de los departamentos de Colombia en los meses de 2020, se detalla que los departamentos con mayor incidencia de casos positivos por COVID-19 fueron San Andrés en marzo 2,019\%, Guainía en septiembre 1,473\%, Bogotá en agosto 1,452\%, Quindío en noviembre 1,329\%, Caquetá en agosto 1,270\% y Risaralda en diciembre 1,157\%.

En la figura 2 se aprecian los datos de la incidencia acumulada (IA) en porcentaje ( $\%$ ), para cada uno de los departamentos de Colombia a 31 de diciembre 2020. Se detalla que los departamentos con mayor IA de casos positivos por COVID-19 fueron Bogotá 6,084\%, Quindío 4,257\%, Antioquía 3,917\%, San Andrés 3,487\%, Caquetá $3,654 \%$ y Atlántico 3,452\% y aquellos con menor IA fueron Arauca 1,555\%, Magdalena 1,493\%, Guajira $1,466 \%$, Cauca $1,254 \%$ y Vichada $1,018 \%$.

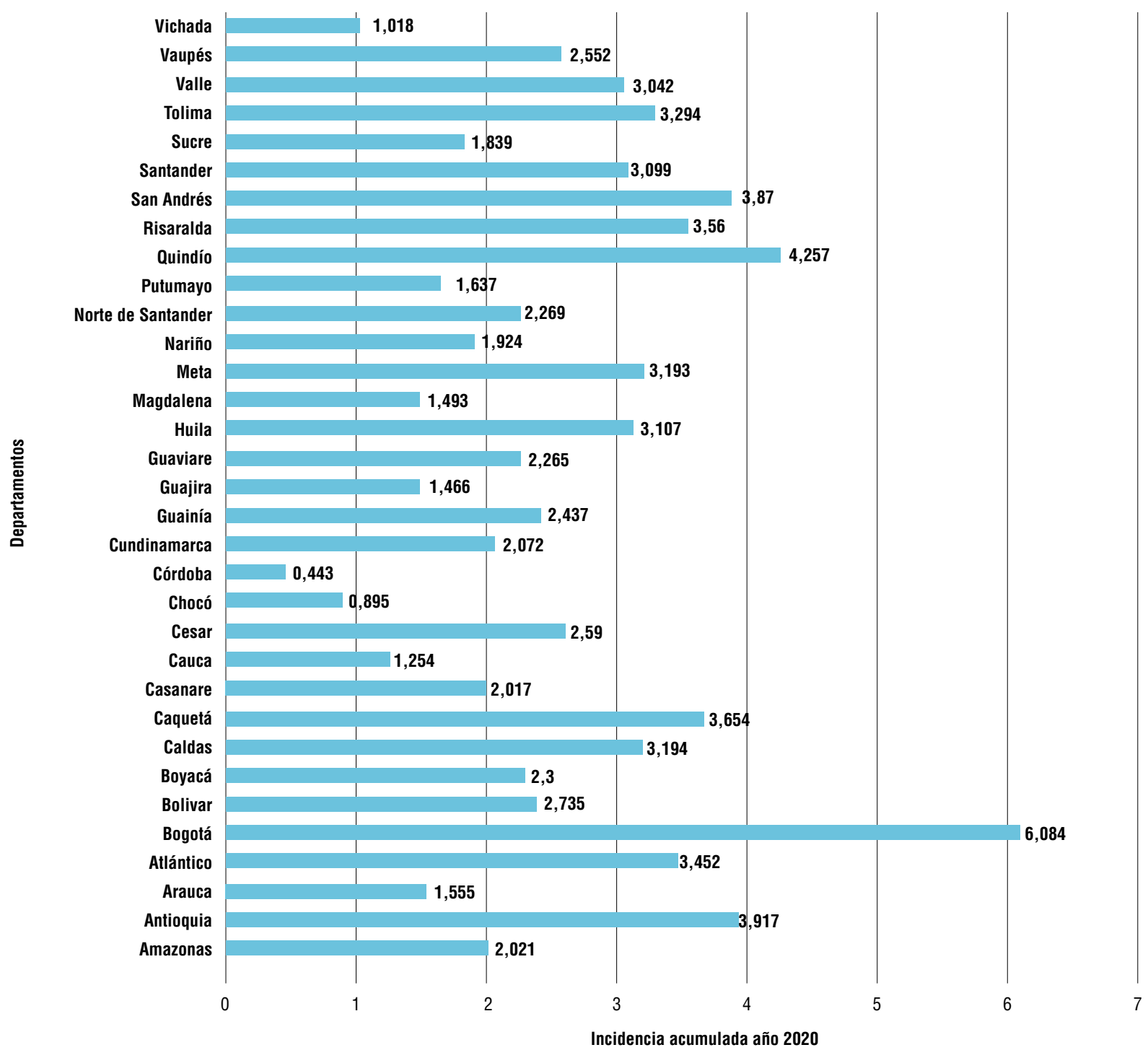

Figura 2. Incidencia acumulada por departamentos en Colombia por COVID-19 para el año 2020. Fuente: El autor. 
CONCLUSIÓN

Se estimó a 31 de diciembre 2020 la incidencia para cada una de los meses de 2020 y se determinó que los de mayor incidencia de casos positivos por COVID-19 fueron diciembre $0,664 \%$, agosto $0,638 \%$, octubre $0,492 \%$ y septiembre $0,431 \%$; los de menor incidencia fueron julio 0,393\%, junio 0,135\%, mayo 0,04\%, abril 0,011\% y marzo $0,001 \%$.

Se estimó los datos de la incidencia en porcentaje (\%) para cada uno de los departamentos de Colombia y meses de 2020, se detalla en los de mayor incidencia de casos positivos por COVID-19; San Andrés en marzo 2,019\%, Guainía en septiembre 1,473\%, Bogotá en agosto 1,452\% y diciembre 1,317\%, Quindío en noviembre 1,329\% y diciembre $1,216 \%$, Caquetá en agosto 1,270\% y Risaralda en diciembre $1,157 \%$.

Se puntualizó que los departamentos con mayor incidencia acumulada (IA) de casos positivos por COVID-19 fueron Bogotá 6,084\%, Quindío 4,257\%, Antioquía 3,917\%, San Andrés 3,487\%, Caquetá 3,654\% y Atlántico 3,452\%; aquellos con menor IA fueron Arauca 1,555\%, Magdalena 1,493\%, Guajira 1,466\%, Cauca 1,254\% y Vichada 1,018\%.

Es importante estudiar la dinámica de posibles brotes infecciosos del COVID-19 en nuestro país, ya que esta enfermedad recientemente surgida ha tenido un rápido crecimiento contagioso. ${ }^{20}$ Es así que en Colombia la curva de casos nuevos diarios, que ha contribuido para explicar la curva epidemiológica del país, ya está por arriba de la primera acometida del virus. El 19 de agosto, durante el primer pico, fue el máximo reportado con 13.056 casos. Ahora, este segundo pico de la enfermedad en el mes de enero registró un récord de contagios diarios de 18.221 casos para el día 8 de enero 2021 y al 11 de enero 2021, se han registrado 1'801.903 de casos nuevos por COVID-19 confirmados y una proporción de casos positivos de 0,23.

Afín con Chaccour citado por Díaz ${ }^{21}$, las múltiples incertidumbres a nivel biológico, clínico y epidemiológico persisten en relación con este nuevo virus. Lo que ya es indudable es que cada país ha examinado o está reconociendo a la misma conminación con disímiles medidas y/o con una mitigación diferente.

\section{REFERENCIAS}

1. Hui DS, E IA, Madani TA, Ntoumi F, Kock R, Dar O, et al. The continuing 2019-nCoV epidemic threat of novel coronaviruses to global health - The latest 2019 novel coronavirus outbreak in Wuhan, China. International journal of infectious diseases : IJID : official publication of the International Society for Infectious Diseases. 2020;91:264-6. Doi: 10.1016/j.ijid.2020.01.009
2. Wu F, Zhao S, Yu B, Chen YM, Wang W, Song ZG, et al. A new coronavirus associated with human respiratory disease in China. Nature. 2020;579(7798):265-9. Doi: 10.1038/s41586-020-2008-3

3. Chan JF-W, Yuan S, Kok K-H, To KK-W, Chu H, Yang J, et al. A familial cluster of pneumonia associated with the 2019 novel coronavirus indicating person-to-person transmission: a study of a family cluster. The Lancet. 2020;395(10223):514-23. Doi: 10.1016/ S0140-6736(20)30154-9

4. Dong E, Du H, Gardner L. An interactive web-based dashboard to track COVID-19 in real time. The Lancet Infectious diseases. 2020;20(5):533-4. Doi: 10.1016/S1473-3099(20)30120-1

5. Guan WJ, Ni ZY, Hu Y, Liang WH, Ou CQ, He JX, et al. Clinical Characteristics of Coronavirus Disease 2019 in China. The New England journal of medicine. 2020;382(18):1708-20. Doi: 10.1056 / NEJMoa2002032

6. Wu Z, McGoogan JM. Characteristics of and Important Lessons From the Coronavirus Disease 2019 (COVID-19) Outbreak in China: Summary of a Report of 72314 Cases From the Chinese Center for Disease Control and Prevention. Jama. 2020;323(13):1239-42. Doi:10.1001/jama.2020.2648

7. Grasselli G, Zangrillo A, Zanella A, Antonelli M, Cabrini L, Castelli A, et al. Baseline Characteristics and Outcomes of 1591 Patients Infected With SARS-CoV-2 Admitted to ICUs of the Lombardy Region, Italy. Jama. 2020;323(16):1574-81. Doi:10.1001/ jama.2020.5394

8. To KK, Tsang OT, Leung WS, Tam AR, Wu TC, Lung DC, et al. Temporal profiles of viral load in posterior oropharyngeal saliva samples and serum antibody responses during infection by SARSCoV-2: an observational cohort study. The Lancet Infectious diseases. 2020;20(5):565-74. Doi: 10.1016/S1473-3099(20)30196-1.

9. Chi X, Yan R, Zhang J, Zhang G, Zhang Y, Hao M, et al. A neutralizing human antibody binds to the N-terminal domain of the Spike protein of SARS-CoV-2. Science. 2020;369(6504):650-5. Doi: 10.1126/science.abc6952

10. van Doremalen N, Lambe $\mathrm{T}$, Spencer A, Belij-Rammerstorfer S, Purushotham JN, Port JR, et al. ChAdOxl nCoV-19 vaccine prevents SARS-CoV-2 pneumonia in rhesus macaques. Nature. 2020;586(7830):578-82. Doi: 10.1038/s41586-020-2608-y

11. Mercado NB, Zahn R, Wegmann F, Loos C, Chandrashekar A, Yu $\mathrm{J}$, et al. Single-shot Ad26 vaccine protects against SARS-CoV-2 in rhesus macaques. Nature. 2020;586(7830):583-8. Doi: 10.1038/ s41586-020-2607-z

12. Díaz-Pinzón J. Uso de modelo predictivo para la dinámica de transmisión del COVID-19 en Colombia. Repert Med Cir. 2020;29(Supl. Núm.1):34-44. Doi: 10.31260/ RepertMedCir.01217372.1056

13. Moreno-Altamirano A, López-Moreno S, Corcho-Berdugo A. Principales medidas en epidemiología. Revista Cubana de Higiene y Epidemiología. 2007;45(1):337-48. 
14. Pita Fernández S, Pértegas Díaz S, Valdés Cañedo F. Medidas de frecuencia de enfermedad [Internet]. España: Elsevier; 2004 [citado 2020 julio 21]; Disponible en: https://www.fisterra. com/formacion/metodologia-investigacion/medidas-frecuenciaenfermedad/.

15. Pinto A. Prevalencia e Incidencia [Internet]. Slideshare; 2014 [citado 2020 julio 21]; Disponible en: https://es.slideshare.net/ alexpinto18/prevalencia-e-incidencia-2?next_slideshow $=2$.

16. Quintana LA. Medidas de frecuencia en epidemiología 2015 [Internet]. Slideshare; 2015 [citado 2020 julio 21]; Disponible en: https://es.slideshare.net/lualberts20/medidas-de-frecuencia-enepidemiologa-2015.

17. Díaz Pinzón JE. Correlación y regresión lineal de la evaluación tiempo y puntaje con recurso interactivo flash. INNOVA Research Journal. 2017;2(10):1-8.
18. Instituto Nacional de Salud. Coronavirus (COVID-2019) en Colombia[Internet] Colombia: Instituto Nacional de Salud; 2020 [cited 2020 noviembre 2]; Available from: https://www.minsalud. gov.co/salud/publica/PET/Paginas/Covid-19_copia.aspx.

19. Departamento Administrativo Nacional de Estadística DANE. Proyecciones y retroproyecciones de población [Internet]. Colombia: DANE; 2000 [citado 2020 julio 25]; Available from: https://www.dane.gov.co/index.php/estadisticas-por-tema/ demografia-y-poblacion/proyecciones-de-poblacion.

20. Díaz Pinzón J. Estudio comparativo entre el contagio durante la cuarentena obligada por el COVID-19 y el contagio durante la apertura gradual y controlada para algunos sectores de la economía en Colombia. Repert Med Cir. 2020;29(Supl.Núm.1):5964. Doi: 10.31260/RepertMedCir.01217372.1073

21. Díaz Pinzón J. Proyección de la propagación del COVID-19 en Colombia. Revista Med. 2020;28(1):11-20. Doi: 10.18359/ rmed.4702 